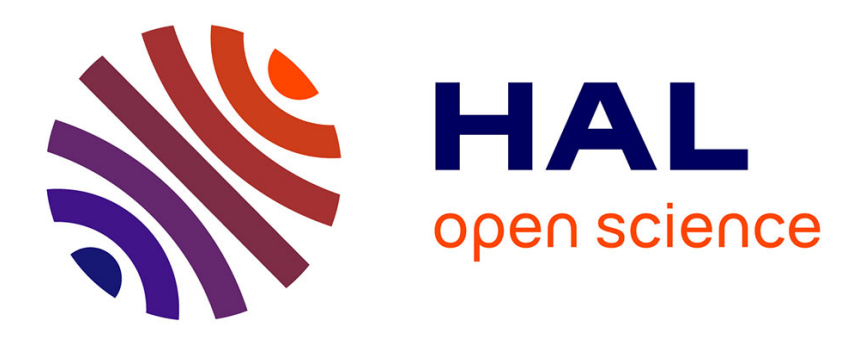

\title{
Optimal prevention of large risks with two types of claims
}

Romain Gauchon, Stéphane Loisel, Jean-Louis Rullière, Julien Trufin

\section{To cite this version:}

Romain Gauchon, Stéphane Loisel, Jean-Louis Rullière, Julien Trufin. Optimal prevention of large risks with two types of claims. Scandinavian Actuarial Journal, In press. hal-02314914v2

\section{HAL Id: hal-02314914 \\ https://hal.science/hal-02314914v2}

Submitted on 29 Oct 2020

HAL is a multi-disciplinary open access archive for the deposit and dissemination of scientific research documents, whether they are published or not. The documents may come from teaching and research institutions in France or abroad, or from public or private research centers.
L'archive ouverte pluridisciplinaire HAL, est destinée au dépôt et à la diffusion de documents scientifiques de niveau recherche, publiés ou non, émanant des établissements d'enseignement et de recherche français ou étrangers, des laboratoires publics ou privés. 


\title{
Optimal prevention of large risks with two types of claims
}

\author{
Romain GAUCHON* \\ Stéphane LOISEL ${ }^{1}$ \\ Jean-Louis RULLIERE ${ }^{1}$
}

Julien TRUFIN ${ }^{2}$

\begin{abstract}
In this paper, we propose and study a risk model with two types of claims in which the insurer may invest into a prevention plan which decreases the intensity of large claims without impacting the small claims. We identify a necessary and sufficient condition for insurers to use prevention if there is no surplus. If, in addition, the severity of large claims dominates that of small claims by the harmonic mean residual life (HMRL) order, insurers invest more in prevention in the presence of a surplus.

Finally, we characterize the asymptotic optimal prevention strategy when the initial surplus tends to infinity in the two main cases where both claim types

*Univ Lyon, Université Claude Bernard Lyon 1, Laboratoire de Sciences Actuarielle et Financière, Institut de Science Financière et d'Assurances (50 Avenue Tony Garnier, F-69007 Lyon, France); addactis in France

Email addresses: romain.gauchon@addactis.com (Romain GAUCHON ),

stephane.loisel@univ-lyon1.fr (Stéphane LOISEL), jean-louis.rulliere@univ-lyon1.fr (Jean-Louis RULLIERE), julien.trufin@ulb.ac.be (Julien TRUFIN)

${ }^{1}$ Univ Lyon, Université Claude Bernard Lyon 1, Laboratoire de Sciences Actuarielle et Financière, Institut de Science Financière et d'Assurances (50 Avenue Tony Garnier, F-69007 Lyon, France)

${ }^{2}$ Université Libre de Bruxelles (ULB), Département de Mathématique, Campus de la Plaine C.P. 210, B-1050 Bruxelles, Belgique.
\end{abstract}


are light-tailed and where one of them is light-tailed and the other one is heavytailed.

Key words: Ruin theory, Prevention, Optimal prevention strategy, Insurance.

\section{Introduction}

Due to their corporate social responsibility, insurance companies tend to be more and more interested in prevention. The South African insurer Discovery has developed the pioneer prevention program Vitality in association with many insurance companies all around the world, such as Generali, John Hancock or Prudential. Based on wearables, these programs aim to offer a premium reduction as an award of an healthy way of life. Similarly, most health companies have launched their own plans, which can take several forms: back-to-work schemes, osteopathy sessions, sports licenses... Some of them, such as the sports related ones, target the global health of policyholders, whereas others, such has psychotherapy sessions or screening campaigns, target more specific and heaviest risks. Since most insurance companies are exposed to multiple risks (e.g. an health insurer is exposed to many diseases), it is therefore interesting to study optimal prevention strategies in presence of two types of claims: large claims for which prevention has an impact and small claims for which the effect of prevention is limited or does not exist. An example of this situation would be the reimbursement of psychotherapy sessions, effectively reducing the risk of relapse (and thus the global frequency) of psychiatric disease, but only affecting marginally the risk of other diseases. Outside of the health sector, one can also think of smoke detectors, only preventing the major risk of fire.

The impact of prevention (sometimes combined with self-insurance, that can be assimilated to reinsurance in our framework) has been investigated in economics by Ehrlich and Becker (1972), Dionne and Eeckhoudt (1985) or Courbage (2001) among others. In ruin theory, a first risk model with prevention has been recently proposed by Gauchon et al. (2020), with a unique set of claims for 
which prevention has some effect. The authors consider an insurance company with initial surplus $U(0)=u$. This company receives premiums at a rate $c$ per unit of time and invests a fixed amount $p$ in prevention per unit of time. The aggregate claim amount up to time $t$ is given by a compound Poisson process $S(t)=\sum_{i=1}^{N(t)} X_{i}$, where $N$ is a Poisson process of arrival intensity $\lambda(p)$ and the $\left(X_{i}\right)_{i \in \mathbb{N}^{*}}$ are i.i.d. random variables, independent from $N$, and with cumulative distribution function $F_{X}$, such that $\mathbb{E}(X)=\mu<\infty$. Gauchon et al. (2020) assume that $\lambda\left(\right.$.) is a decreasing, strictly convex, positive, and $\mathcal{C}^{2}$ function defined on $[0, c]$. They determine the optimal prevention investment for different risk indicators, and in particular for the ruin probability. This optimal prevention strategy does not depend on the initial surplus level.

Most economic models of prevention built on Ehrlich and Becker (1972) involve a single source of risk and a single type of prevention activity. Exceptions include Courbage and Rey (2012) who consider an independent background risk, Hofmann and Peter (2015) who study the interplay of early and late prevention, and Courbage et al. (2017) who investigate prevention of correlated risks. When it comes to prevention by insurers, Gauchon et al. (2020) focus on a single source of risk. We instead distinguish between large claims and small claims and assume that prevention reduces only the intensity of large claims. We show that in this case, the optimal prevention investment does depend on the initial surplus level. One could think that it would be optimal to do less prevention if one has a high initial surplus level. In the present paper, we show that it is the opposite: when the initial surplus is large enough, under reasonable conditions, optimising the ruin probability leads to implement more prevention than without an initial surplus.

Our main contribution is threefold. First, we propose and analyse a first risk model with two types of claims, where prevention has some impact on the large claims only. Second, we show that prevention is advantageous when claim severities of small claims and large claims are ordered in the sense of the so-called Harmonic Mean Residual Lifetime (HMRL) order, and that the optimal preven- 
tion effort is higher with a positive initial surplus than when the initial surplus is zero. Third, we characterize the asymptotic optimal prevention strategy when the initial surplus tends to infinity in the two main cases where both claim types are light-tailed and where one of them is light-tailed and the other one is heavytailed. In addition, on the occasion of a natural example of the second case, we provide a necessary and sufficient condition to order a translated Exponential random variable and a Pareto one in the HMRL order.

The paper is organized as follows. In Section 2, we describe our model with two types of claims. In Section 3, we recall the definition of the HMRL order and we notably provide a necessary and sufficient condition to order a translated Exponential random variable and a Pareto one in the HMRL order. In Section 4, we show that, under some HMRL ordering condition, the optimal prevention level is positive and higher than when the initial surplus is zero. In Section 5, we study the asymptotic optimal prevention strategy when both claim types are light tailed. Section 6 is devoted to the case where the second type of claims is heavy tailed.

\section{The model}

Let us consider an insurer facing two types of claims, namely small claims and large claims. The insurer invests an amount $p \in[0, c]$ in preventior ${ }^{3}$, in order to prevent large claims from happening. In such a case, the number of claims $N(t)$ up to time $t$ can be written as $N_{1}(t)+N_{2}(t)$, where $N_{1}$ (resp. $N_{2}$ ) is a Poisson process of arrival intensity $\lambda_{1}$ (resp. $\lambda_{2}(p)$ ) that represents the number of small claims (resp. large claims), such that $\lambda(p)=\lambda_{1}+\lambda_{2}(p)$. The function $\lambda_{2}(p)$ is assumed to be a decreasing, strictly convex, positive, and $\mathcal{C}^{2}$ function defined on $[0, c]$. These constraints on $\lambda_{2}($.$) are similar to the ones imposed on \lambda($.$) in$ Gauchon et al. (2020) and we refer the reader to this latter paper for a discussion

\footnotetext{
${ }^{3}$ For the following, prevention refers to self-protection, in the sense of Ehrlich and Becker
} $(1972)$. 
about these assumptions. The Poisson processes $N_{1}$ and $N_{2}$ are assumed to be independent. Hence, the aggregate claim amount $S(t)=\sum_{k=1}^{N(t)} X_{k}$ up to time $t$ can be decomposed as

$$
S(t)=\sum_{k=1}^{N(t)} X_{k}=\sum_{i=1}^{N_{1}(t)} X_{i}^{(1)}+\sum_{j=1}^{N_{2}(t)} X_{j}^{(2)},
$$

where the $X_{i}^{(1)} \mathrm{s}\left(\right.$ resp. $X_{j}^{(2)} \mathrm{s}$ ) are the claim severities for small claims (resp. large claims) and are assumed to be independent and identically distributed as $X^{(1)}$ (resp. $\left.X^{(2)}\right)$ with mean $\mu_{1}$ (resp. $\mu_{2}$ ) such that $\mu_{1} \leq \mu_{2}$. Furthermore, the claim severities $X_{i}^{(1)}$ and $X_{j}^{(2)}$ are also supposed to be independent and independent of the number of claims $N_{1}(t)$ and $N_{2}(t)$. Such a model amounts to consider the claim severity $X_{k}$ with cumulative distribution function

$$
F_{X}(x)=\frac{\lambda_{1} F_{X^{(1)}}(x)+\lambda_{2}(p) F_{X^{(2)}}(x)}{\lambda(p)}, \quad x \geq 0,
$$

where $F_{X^{(1)}}($.$) and F_{X^{(2)}}($.$) are the cumulative distribution functions of X^{(1)}$ and $X^{(2)}$, respectively.

In the following, we consider an insurer with a surplus process given by

$$
\begin{aligned}
U(t, p) & =u+(c-p) t-\sum_{k=1}^{N(t)} X_{k} \\
& =u+(c-p) t-\sum_{i=1}^{N_{1}(t)} X_{i}^{(1)}-\sum_{j=1}^{N_{2}(t)} X_{j}^{(2)} .
\end{aligned}
$$

In order to avoid ruin with certainty, we define $p_{l i m} \in[0, c]$ as the solution of

$$
\frac{\lambda_{1} \mu_{1}+\lambda_{2}\left(p_{\text {lim }}\right) \mu_{2}}{c-p_{\text {lim }}}=1
$$

and we require $p<p_{\text {lim }}$. Since functions $\lambda_{2}, h: p \mapsto \frac{\lambda_{1} \mu_{1}+\lambda_{2}(p) \mu_{2}}{c-p}$ and derivatives of $h$ follow similar properties as the ones in Proposition 1 of Gauchon et al. (2020), it is possible to show that the function $h$ is either increasing or first decreasing and then increasing with $p$. Thus, the intermediate value theorem ensures the existence and the uniqueness of $p_{\text {lim }}$ when $\frac{\lambda_{1} \mu_{1}+\lambda_{2}(0) \mu_{2}}{c}<1$, which is assumed in the rest of the paper. 
Remark: Since the function $h$ is either increasing or first decreasing and then increasing with $p$, we have $\frac{\partial h}{\partial p}\left(p_{\text {lim }}\right)>0$. Thus, the implicit function theorem gives easily comparative statics of $p_{l i m}$ as a function of exogenous parameters : $\frac{\partial p_{l i m}}{\partial \lambda_{1}}<0, \frac{\partial p_{l i m}}{\partial \mu_{1}}<0, \frac{\partial p_{l i m}}{\partial \mu_{2}}<0$ and $\frac{\partial p_{l i m}}{\partial c}>0$.

In this model, prevention does not only act on the number of claims but also on the distribution $F_{X}$ of the claim severities $X_{k}$. In the following, we denote by $X(p)$ the random variable $X$ to make explicit the link with the prevention amount $p$.

In this paper, we are interested in the prevention amount $p^{*}(u)$ that minimizes the ruin probability

$$
\psi(u, p)=\mathbb{P}(\exists t>0 \text { such that } U(t, p)<0) .
$$

It is known that $\psi(u, p)$ coincides with the tail function of a compound geometric distribution, namely

$$
\psi(u, p)=\mathbb{P}\left(\sum_{j=1}^{M} D_{j}>u\right),
$$

where $M$ follows the geometric distribution with success probability $\varphi(0, p)=$ $1-\psi(0, p)$. The random variables $D_{1}, D_{2}, \ldots$ are called ladder heights and are independent and identically distributed as the random variable $D$, also denoted $D(p)$ in the following to make explicit the dependence with respect to $p$. In our compound Poisson model, the cumulative distribution function of $D(p)$ is given by the integrated tail distribution

$$
F_{D(p)}(u)=\int_{0}^{u} \frac{1-F_{X}(x)}{\mathbb{E}(X)} d x, \quad u>0 .
$$

From (4), we get the Pollaczeck Khinchin formula

$$
\varphi(u, p)=\varphi(0, p) \sum_{n=0}^{\infty}(1-\varphi(0, p))^{n} F_{D(p)}^{* n}(u),
$$

where $F_{D(p)}^{* n}$ is the $n$-fold convolution of $F_{D(p)}$, and $\varphi(0, p)=1-\frac{\lambda_{1} \mu_{1}+\lambda_{2}(p) \mu_{2}}{c-p}$. This formula guarantees the existence of the derivative $\frac{\partial \varphi(u, p)}{\partial p}$ when $\frac{\partial F_{D(p)}(u)}{\partial p}$ exists. 
Denoting by $M_{X}($.$) the moment generating function of X$, we can define for our model the adjustment coefficient $\kappa(p)$, which verifies

$$
\lambda(p)+(c-p) \kappa(p)=\lambda(p) M_{X}(\kappa(p)) .
$$

It always exists when

$$
M_{X}(s)<\infty \text { for all } s \in \mathbb{R}
$$

or when there exists $s^{*}>0$ such that

$$
M_{X}(s)<\infty \quad \text { for all } s<s^{*} \quad \text { and } \quad M_{X}(s)=\infty \text { for all } s \geq s^{*} .
$$

We refer the reader to Asmussen and Albrecher (2010) for more details.

Models with multiple risks have been introduced by Cramér (1955) and have been used for example by Dickson and Gray (1984), Bowers et al. (1984) or Gerber et al. (1987).

\section{Preliminaries}

Before studying the optimal prevention amount $p^{*}(u)$, we state the Harmonic Mean Residual Life (HMRL) order (more details can be found in Shaked and Shanthikumar (2007)). Also, we prove two results that will be useful in the subsequent analysis.

Definition 1. Given two non-negative random variables $X^{(1)}$ and $X^{(2)}$ with respective cumulative distribution functions $F_{X^{(1)}}$ and $F_{X^{(2)}}, X^{(1)}$ is said to be smaller than $X^{(2)}$ in the harmonic mean residual life order (denoted as $X^{(1)} \preceq_{\text {hmrl }} X^{(2)}$ ) when

$$
\frac{\int_{t}^{\infty} \bar{F}_{X^{(1)}}(u) d u}{\mathbb{E}\left(X^{(1)}\right)} \leq \frac{\int_{t}^{\infty} \bar{F}_{X^{(2)}}(u) d u}{\mathbb{E}\left(X^{(2)}\right)} \quad \text { for all } t \geq 0
$$

where $\bar{F}_{X^{(1)}}=1-F_{X^{(1)}}$ and $\bar{F}_{X^{(2)}}=1-F_{X^{(2)}}$. The inequality in 10 can be equivalently written as

$$
\frac{\mathbb{E}\left(\left(X^{(1)}-t\right)_{+}\right)}{\mathbb{E}\left(X^{(1)}\right)} \leq \frac{\mathbb{E}\left(\left(X^{(2)}-t\right)_{+}\right)}{\mathbb{E}\left(X^{(2)}\right)} \quad \text { for all } t \geq 0 .
$$


The mean residual life of a random variable $X$ at $t$ is defined as $m(t)=\mathbb{E}(X-$ $t \mid X>t)$ for $t<t^{*}$ and $m(t)=0$ otherwise, where $t^{*}=\sup \left\{t: 1-F_{X}(t)>0\right\}$. Let $X^{(1)}$ and $X^{(2)}$ be two non-negative random variables. Then, denoting by $m^{(1)}$ (resp. $\left.m^{(2)}\right)$ the mean residual life of $X^{(1)}$ (resp. $X^{(2)}$ ), it can be shown that $X^{(1)} \preceq_{\mathrm{hmrl}} X^{(2)}$ if and only if the harmonic averages of $m^{(1)}$ and $m^{(2)}$ satisfy

$$
\left[\frac{1}{x} \int_{0}^{x} \frac{1}{m^{(1)}(u)} d u\right] \leq\left[\frac{1}{x} \int_{0}^{x} \frac{1}{m^{(2)}(u)} d u\right]
$$

for all $x>0$. Therefore, ordering two random variables by the HMRL order is equivalent to ordering the harmonic averages of their corresponding mean residual life.

Below we give some examples taken from Heilmann and Schröter (1991) where $X^{(1)} \preceq_{\text {hmrl }} X^{(2)}$ holds true:

- If $X^{(1)}$ is Uniformly distributed over the interval $[a, b]$ and $X^{(2)}$ is Uniformly distributed over the interval $\left(a^{\prime}, b^{\prime}\right)$, then $X^{(1)} \preceq_{\mathrm{hmrl}} X^{(2)}$ if and only if $a+b \leq a^{\prime}+b^{\prime}$ and $b \leq b^{\prime}$.

- If $X^{(1)}$ is Exponentially distributed with mean $1 / a$ and $X^{(2)}$ is Exponentially distributed with mean $1 / a^{\prime}$ then $X^{(1)} \preceq_{\mathrm{hmrl}} X^{(2)}$ if and only if $a \geq a^{\prime}$.

- If $X^{(1)}$ is Pareto distributed with parameters $a$ and $b$, that is, $X^{(1)}$ has distribution function $1-\left(\frac{a}{x}\right)^{b}, x>a$, and if $X^{(2)}$ is Pareto distributed with parameters $a^{\prime}$ and $b^{\prime}$ with $\min \left(b, b^{\prime}\right)>1$, then $X^{(1)} \preceq_{\mathrm{hmrl}} X^{(2)}$ if and only if $\frac{b-1}{b^{\prime}-1} \geq \max \left(\frac{a}{a^{\prime}}, 1\right)$

We will now order two random variables whose distributions belong to different classes.

Proposition 2. If $X^{(1)}$ follows a translated Exponential distribution over the interval $[a, \infty]$ with mean $a+\frac{1}{\lambda}$ and $X^{(2)}$ is Pareto distributed with parameters $a$ and $b$, then $X^{(1)} \preceq_{h m r l} X^{(2)}$ if and only if $b \leq \lambda a+1$. 
Proof. Since

$$
F_{X^{(1)}}(x)=1-e^{-\lambda(x-a)}, \quad x \geq a
$$

and

$$
F_{X^{(2)}}(x)=1-\left(\frac{a}{x}\right)^{b} \quad x \geq a,
$$

inequality 10 is equivalent to

$$
\frac{e^{\lambda(a-t)}}{\lambda a+1} \leq\left(\frac{a}{t}\right)^{b-1} \frac{1}{b}, \quad \text { for all } t \geq a .
$$

In particular, inequality (14) must hold for $t=a$. A necessary condition for $X^{(1)} \preceq_{\mathrm{hmrl}} X^{(2)}$ is thus given by

$$
\frac{1}{\lambda a+1} \leq \frac{1}{b}
$$

Let us now show that 15 is actually a sufficient condition for $X^{(1)} \preceq_{\mathrm{hmrl}} X^{(2)}$. Inequality 14 can be rewritten as

$$
\log \left(\frac{b}{\lambda a+1}\right)+\lambda(a-t)-(b-1)[\log (a)-\log (t)] \leq 0 \quad \text { for all } t \geq a .
$$

Considering $t>a$ and introducing the function $g(t)=\log \left(\frac{b}{\lambda a+1}\right)+\lambda(a-t)-$ $(b-1)[\log (a)-\log (t)]$, we easily see that $g^{\prime}(t)<0$ if and only if $\frac{b-1}{\lambda}<t$, which is always true since 15 yields $\frac{b-1}{\lambda} \leq a<t$. Therefore, $g^{\prime}(t)<0$ for all $t>a$. Now, as 15 implies that $g(a)<0$, we finally get $g(t)<0$ for all $t \geq a$.

Notice that if $b>1, \mathbb{E}\left(X^{(1)}\right) \leq \mathbb{E}\left(X^{(2)}\right)$ if and only if $1+\lambda a \leq b \frac{a \lambda}{b-1}$. Indeed, multiplying both sides of the last inequality by $b-1$ shows that $X^{(1)} \preceq_{\mathrm{hmrl}} X^{(2)}$ is equivalent to $\mathbb{E}\left(X^{(1)}\right) \leq \mathbb{E}\left(X^{(2)}\right)$.

The next proposition will be useful to prove Proposition 5 In particular, it is based on the observation that, in the context of ruin theory, if a claim severity $X^{(1)}$ is smaller than another claim severity $X^{(2)}$ in the sense of the HMRL order, then it means that the ladder height associated to $X^{(1)}$ is smaller than the ladder height associated to $X^{(2)}$ in the sense of the usual stochastic order. Claim severity $X^{(1)}$ is smaller than claim severity $X^{(2)}$ in the usual stochastic order, denoted $X^{(1)} \preceq_{\text {st }} X^{(2)}$, when $F_{X^{(1)}}(t) \geq F_{X^{(2)}}(t)$ for all $t$. 
Proposition 3. Let $p_{1} \leq p_{2}$. We have $X^{(1)} \preceq_{h m r l} X^{(2)}$ if and only if $D\left(p_{2}\right) \preceq_{s t}$ $D\left(p_{1}\right)$.

Proof. We know from Theorem 2.B.2. in Shaked and Shanthikumar (2007) that

$$
X\left(p_{2}\right) \preceq_{\mathrm{hmrl}} X\left(p_{1}\right) \Leftrightarrow D\left(p_{2}\right) \preceq_{\mathrm{st}} D\left(p_{1}\right) .
$$

Moreover, we remark that $\frac{\int_{t}^{\infty} \bar{F}_{X\left(p_{2}\right)}(u) d u}{\mathbb{E}\left(X\left(p_{2}\right)\right)} \leq \frac{\int_{t}^{\infty} \bar{F}_{X\left(p_{1}\right)}(u) d u}{\mathbb{E}\left(X\left(p_{1}\right)\right)}$ is equivalent to

$$
\left(\frac{\lambda_{1} \mu_{1}}{\lambda_{1} \mu_{1}+\lambda_{2}\left(p_{2}\right) \mu_{2}}-\frac{\lambda_{1} \mu_{1}}{\lambda_{1} \mu_{1}+\lambda_{2}\left(p_{1}\right) \mu_{2}}\right)\left(\frac{\int_{t}^{\infty} \bar{F}_{X^{(2)}}(u) d u}{\mu_{2}}-\frac{\int_{t}^{\infty} \bar{F}_{X^{(1)}}(u) d u}{\mu_{1}}\right) \geq 0,
$$

which, in turn, is equivalent to the left-hand side of Equation (17).

\section{Optimal prevention amount $p^{*}(u)$}

Let us first consider an insurer with no initial surplus. We then have the following result.

Proposition 4. The optimal prevention amount $p^{*}(0)$ is positive if and only if

$$
-\lambda_{2}^{\prime}(0)>\frac{\lambda_{1} \mu_{1}+\lambda_{2}(0) \mu_{2}}{\mu_{2} c} .
$$

In this case, $p^{*}(0)$ satisfies

$$
-\lambda_{2}^{\prime}\left(p^{*}(0)\right)=\frac{\lambda_{1} \mu_{1}+\lambda_{2}\left(p^{*}(0)\right) \mu_{2}}{\mu_{2}\left(c-p^{*}(0)\right)} .
$$

Proof. Starting from

$$
\varphi(0, p)=1-\frac{\mu_{1} \lambda_{1}+\mu_{2} \lambda_{2}(p)}{c-p}
$$

we get

$$
\frac{\partial \varphi(0, p)}{\partial p}=-\frac{\lambda_{1} \mu_{1}}{(c-p)^{2}}-\frac{\lambda_{2}(p) \mu_{2}}{(c-p)^{2}}-\frac{\lambda_{2}^{\prime}(p) \mu_{2}}{c-p}
$$

and

$$
\frac{\partial^{2} \varphi(u, p)}{\partial p^{2}}=\frac{2}{c-p} \frac{\partial \varphi(0, p)}{\partial p}-\frac{\lambda_{2}^{\prime \prime}(p) \mu_{2}}{c-p} .
$$


Now, similarly to the proof of Proposition 1 in Gauchon et al. (2020), it suffices to see that Equations 22 and 23 enable us to prove that if $\frac{\partial \varphi(0,0)}{\partial p} \leq 0$, then $\frac{\partial \varphi(0, p)}{\partial p}<0$ and $\frac{\partial^{2} \varphi(0, p)}{\partial p^{2}}<0$ for all $p>0$.

Hence, if Condition $\sqrt{19}$ is fulfilled, an insurer with no initial surplus can decrease its risk by investing a part of its premiums in prevention.

Condition (19) is more easily met than Condition (10) in Gauchon et al. (2020), which suggests that, in some cases, insurers will optimally spend money in prevention by targeting only large claims while they should not make any expenses for prevention if they would target both small and large claims.

Remark: The implicit function theorem allows us to write $p^{*}(0)$ as a function of $c, \lambda_{1}, \mu_{1}$ and $\mu_{2}$, so that one can study the sensitivity of $p^{*}(0)$ with respect to each of these parameters. For instance, rewriting Equation 20 as

$$
p^{*}(0)=c+\frac{\lambda_{1} \mu_{1}+\lambda_{2}\left(p^{*}(0)\right) \mu_{2}}{\lambda_{2}^{\prime}\left(p^{*}(0)\right) \mu_{2}}
$$

we get

$$
\frac{\partial p^{*}(0)}{\partial \mu_{1}}=\frac{\lambda_{1} \lambda_{2}^{\prime}\left(p^{*}(0)\right) \mu_{2}}{\left[\lambda_{1} \mu_{1}+\lambda_{2}\left(p^{*}(0)\right) \mu_{2}\right] \lambda_{2}^{\prime \prime}\left(p^{*}(0)\right) \mu_{2}} .
$$

Since $\lambda_{2}$ is a decreasing convex function, we have $\frac{\partial p^{*}(0)}{\partial \mu_{1}} \leq 0$. Similarly, one can show that $\frac{\partial p^{*}(0)}{\partial \lambda_{1}} \leq 0, \frac{\partial p^{*}(0)}{\partial c} \geq 0$ and $\frac{\partial p^{*}(0)}{\partial \mu_{2}} \geq 0$. Thus, the higher the average severity of large claims (resp. small claims), the more (resp. the less) insurers should invest in prevention. It shows that $p^{*}(0)$ is influenced by the relative difference in average severity between small and large claims.

Next to Condition (19), the following result gives an additional sufficient condition ensuring that an insurer with a positive initial surplus can also decrease its risk through prevention.

Proposition 5. If Condition (19) is verified and if $X^{(1)} \preceq_{h m r l} X^{(2)}$, then $p^{*}(u)>$ 0 for all $u \geq 0$. 
Proof. By definition, $\varphi\left(u, p_{l i m}\right)=0$ and $p<p_{\text {lim }}$ ensures that $\varphi(u, 0)>0$. As Condition 19 holds true, we know from the boundedness theorem that $\varphi(u,$. reaches a maximum for one $p \in\left[0, p_{\text {lim }}\right]$.

Now, it suffices to prove that $\frac{\partial \varphi(0, p)}{\partial p}>0$ implies $\frac{\partial \varphi(u, p)}{\partial p}>0$ and the proof will be completed. From the Pollaczeck Khinchin formula (6), we get

$$
\begin{aligned}
\frac{\partial \varphi(u, p)}{\partial p}= & \frac{\partial \varphi(0, p)}{\partial p} \sum_{n=0}^{\infty}(1-\varphi(0, p))^{n} F_{D(p)}^{* n}(u)\left(1-\frac{\varphi(0, p)}{1-\varphi(0, p)} n\right) \\
& +\varphi(0, p) \sum_{n=0}^{\infty}(1-\varphi(0, p))^{n} \frac{\partial F_{D(p)}^{* n}(u)}{\partial p} .
\end{aligned}
$$

First, we show that

$$
\sum_{n=0}^{\infty}(1-\varphi(0, p))^{n} F_{D(p)}^{* n}(u)\left(1-\frac{\varphi(0, p)}{1-\varphi(0, p)} n\right) \geq 0 .
$$

Let $v_{n}=(1-\varphi(0, p))^{n}\left(1-\frac{\varphi(0, p)}{1-\varphi(0, p)} n\right), n \in \mathbb{N}$. We get

$$
\begin{aligned}
\sum_{n=0}^{\infty} v_{n} & =\sum_{n=0}^{\infty}(1-\varphi(0, p))^{n}\left(1+n-\frac{n}{1-\varphi(0, p)}\right) \\
& =\sum_{n=0}^{\infty}(1-\varphi(0, p))^{n}(1+n)-\sum_{n=0}^{\infty}(1-\varphi(0, p))^{n-1} n \\
& =0 .
\end{aligned}
$$

Also, for all $n \in \mathbb{N}$, we have

$$
v_{n+1}-v_{n}=(1-\varphi(0, p))^{n-1} \varphi(0, p)[\varphi(0, p)(n+2)-2] .
$$

Thus, the sequence $\left\{v_{n}, n \in \mathbb{N}\right\}$ is first decreasing and then increasing. Since $\sum_{n=0}^{\infty} v_{n}=0$, there exists $K \in \mathbb{N}^{*}$ such that $v_{n} \geq 0$ for $n \leq K$ and $v_{n} \leq 0$ for $n>K$. Moreover, we notice that $F_{D(p)}^{* n+1}(u) \leq F_{D(p)}^{* n}(u) \leq 1$ for all $n \in \mathbb{N}^{*}$. Hence,

$$
\begin{aligned}
\sum_{n=0}^{\infty} v_{n} F_{D(p)}^{* n}(u) & =\sum_{n=0}^{K} v_{n} F_{D(p)}^{* n}(u)+\sum_{n=K+1}^{\infty} v_{n} F_{D(p)}^{* n}(u) \\
& \geq \sum_{n=0}^{K} v_{n} F_{D(p)}^{* K}(u)+\sum_{n=K+1}^{\infty} v_{n} F_{D(p)}^{* K}(u) \\
& \geq 0
\end{aligned}
$$


so that

$$
\sum_{n=0}^{\infty}(1-\varphi(0, p))^{n} F_{D(p)}^{* n}(u)\left(1-\frac{\varphi(0, p)}{1-\varphi(0, p)} n\right) \geq 0 .
$$

Secondly, we find

$$
\varphi(0, p) \sum_{n=0}^{\infty}(1-\varphi(0, p))^{n} \frac{\partial F_{D(p)}^{* n}(u)}{\partial p} \geq 0
$$

Indeed, as the usual stochastic order is closed under convolution, Proposition 3 directly leads to $\frac{\partial F_{D(p)}^{* n}(u)}{\partial p} \geq 0$, which completes the proof.

Thus, in case 190 and $X^{(1)} \preceq_{\mathrm{hmrl}} X^{(2)}$ hold true, we know that $p^{*}(u)>0$ for all $u$. We learn from the next proposition that the minimum of $p^{*}(u)$ is reached in $u=0$. Notice that contrary to Gauchon et al. (2020), one observes that $p^{*}(u)$ is not constant anymore in the present context.

Proposition 6. If (19) holds true and if we have $X^{(1)} \preceq_{h m r l} X^{(2)}$, then $p^{*}(0)<$ $p^{*}(u)$ for all $u>0$.

Proof. From Equation 26) and inequalities 29) and (30), one sees that $\frac{\partial \varphi\left(0, p^{*}(u)\right)}{\partial p}<$ 0 is a necessary condition for $\frac{\partial \varphi\left(u, p^{*}(u)\right)}{\partial p}=0$. Now, Equations 22 and $23 \mathrm{im}-$ ply that $\varphi(0,$.$) is increasing on \left[0, p^{*}(0)\right]$ and decreasing on $\left[p^{*}(0), p_{\text {lim }}\right]$. Thus, $\frac{\partial \varphi\left(0, p^{*}(u)\right)}{\partial p}<0$ is only possible when $p^{*}(u)>p^{*}(0)$.

Propositions 7 and 8 will show that the asymptotic values for $p^{*}($.$) are indeed$ larger than $p^{*}(0)$. This suggests that $p^{*}($.$) is increasing with u$ but we are not in a position to prove this claim.

An explanation of Proposition 6 could be that, when the reserves are huge, one knows that ruin is most likely to occur due to an extreme claim so that small claims play a less important role. However, when the initial surplus is zero, the insurer must escape the dangerous zone as fast as possible. Because this time small claims matter, the insurer cannot afford to invest in prevention as much as when the surplus is large. This is in line with the remark following Proposition 4 when $u=0$, the larger is the relative importance of large claims compared to small claims, the more the insurer should spend money in prevention. 


\section{Optimal prevention amount when the adjustment coefficient exists for all $p$}

In this section, we consider claim severities $X^{(1)}$ and $X^{(2)}$ such that the adjustment coefficient $\kappa(p)$ exists for all $p \in[0, c]$. This requirement is for example met when both random variables $X^{(1)}$ and $X^{(2)}$ are Exponentially distributed, which is a particular case of models considered in Gerber et al. (1987) and Dufresne and Gerber (1988).

When the initial surplus $u$ goes to infinity, we show in the next proposition that

the optimal prevention amount $p^{*}(u)$ converges to the one that maximizes the adjustment coefficient $\kappa(p)$, denoted $p_{\kappa}^{*}$.

Proposition 7. The prevention amount $p_{\kappa}^{*}$ maximizing the adjustment coefficient $\kappa(p)$ is solution of the equation

$$
-\lambda_{2}^{\prime}(p)\left[(c-p)+\lambda_{1} \mathbb{E}\left(e^{\kappa(p) X(p)}\left(X^{(1)}-X^{(2)}\right)\right)\right]=\lambda_{1}+\lambda_{2}(p) .
$$

Moreover, we have

$$
\lim _{u \rightarrow \infty} p^{*}(u)=p_{\kappa}^{*}
$$

Proof. The adjustment coefficient $\kappa(p)$ verifies

$$
\lambda_{1}+\lambda_{2}(p)+(c-p) \kappa(p)=\left(\lambda_{1}+\lambda_{2}(p)\right) M_{X(p)}(\kappa(p)) .
$$

The implicit function theorem shows that $\kappa($.$) is differentiable. Now, deriving$ (33) with respect to $p$ yields

$$
\begin{aligned}
\lambda_{2}^{\prime}(p)\left(1-M_{X}(\kappa(p))\right)-\kappa(p)= & \left(\lambda_{1}+\lambda_{2}(p)\right)\left(\frac{\lambda_{1} \kappa(p)}{\lambda_{1}+\lambda_{2}(p)}\right)^{\prime} M_{X^{(1)}}^{\prime}\left(\frac{\lambda_{1} \kappa(p)}{\lambda_{1}+\lambda_{2}(p)}\right) M_{X^{(2)}}\left(\frac{\lambda_{2}(p) \kappa(p)}{\lambda_{1}+\lambda_{2}(p)}\right) \\
& +\left(\lambda_{1}+\lambda_{2}(p)\right)\left(\frac{\lambda_{2}(p) \kappa(p)}{\lambda_{1}+\lambda_{2}(p)}\right)^{\prime} M_{X^{(1)}}\left(\frac{\lambda_{1} \kappa(p)}{\lambda_{1}+\lambda_{2}(p)}\right) M_{X^{(2)}}^{\prime}\left(\frac{\lambda_{2}(p) \kappa(p)}{\lambda_{1}+\lambda_{2}(p)}\right) .
\end{aligned}
$$

Now, Equation 33 can be rewritten as

$$
\left(M_{X}(\kappa(p))-1\right)=\frac{(c-p) \kappa(p)}{\lambda_{1}+\lambda_{2}(p)} .
$$


So, combining (34) and (35) and taking $p=p_{\kappa}^{*}$, we get

$$
-\lambda_{2}^{\prime}\left(p_{\kappa}^{*}\right)\left[\left(c-p_{\kappa}^{*}\right)+\lambda_{1} \mathbb{E}\left(e^{\kappa\left(p_{\kappa}^{*}\right) X\left(p_{\kappa}^{*}\right)}\left(X^{(1)}-X^{(2)}\right)\right)\right]=\lambda_{1}+\lambda_{2}\left(p_{\kappa}^{*}\right)
$$

since $\kappa^{\prime}\left(p_{\kappa}^{*}\right)=0$. Notice that Equation (36) admits a unique solution when Condition 19 holds true.

Let us now prove that $\lim _{u \rightarrow \infty} p^{*}(u)=p_{\kappa}^{*}$. It is known that $\varphi(u, p)$ can be written as

$$
\varphi(u, p)=1-e^{-\kappa(p) u} \mathbb{E}_{\kappa(p)}\left(e^{-\kappa(p) \xi(u)}\right),
$$

where $\xi(u)$ is the random variable representing the overshoot in case of ruin and $\mathbb{E}_{\kappa(p)}$ is the expected value computed under a change of probability measure using the exponentials families (for more details, we refer the reader to Section 4.4 and Equations (5.4) to (5.6) in Section 4.5 of Asmussen and Albrecher (2010)). Thus, for all $u>0$, we have

$$
\begin{aligned}
\frac{\partial \varphi\left(u, p^{*}(u)\right)}{\partial p}= & -\kappa^{\prime}\left(p^{*}(u)\right) u e^{-\kappa\left(p^{*}(u)\right) u} \mathbb{E}_{\kappa(p)}\left(e^{-\kappa\left(p^{*}(u)\right) \xi(u)}\right) \\
& +e^{-\kappa\left(p^{*}(u)\right) u} \frac{\partial \mathbb{E}_{\kappa(p)}\left(e^{-\kappa\left(p^{*}(u)\right) \xi(u)}\right)}{\partial p} \\
= & 0,
\end{aligned}
$$

from which we deduce

$$
\frac{\frac{\partial \mathbb{E}_{\kappa(p)}\left(e^{-\kappa\left(p^{*}(u)\right) \xi(u)}\right)}{\partial p}}{u \mathbb{E}_{\kappa(p)}\left(e^{-\kappa\left(p^{*}(u)\right) \xi(u)}\right)}=\kappa^{\prime}\left(p^{*}(u)\right) .
$$

Moreover, the Cramer-Lundberg approximation shows that

$$
\lim _{u \rightarrow \infty} \mathbb{E}_{\kappa(p)}\left(e^{-\kappa(p) \xi(u)}\right)=C(p)=(c-p) \frac{\varphi(0, p)}{\left(\lambda_{1}+\lambda_{2}(p)\right) M_{X}^{\prime}(\kappa(p))-c+p}>0 .
$$

Now, since $p$ is bounded, $C(p)$ is bounded as well. Furthermore, we get

$$
\lim _{u \rightarrow \infty} \frac{\partial \mathbb{E}_{\kappa(p)}\left(e^{-\kappa\left(p_{\kappa}^{*}\right) \xi(u)}\right)}{\partial p}=C^{\prime}\left(p_{\kappa}^{*}\right) \in \mathbb{R} .
$$

Then, combining (40) and (41) leads to

$$
\lim _{u \rightarrow \infty} \frac{\frac{\partial \mathbb{E}_{\kappa(p)}\left(e^{-\kappa\left(p^{*}(u)\right) \xi(u)}\right)}{\partial p}}{u \mathbb{E}_{\kappa(p)}\left(e^{-\kappa\left(p^{*}(u)\right) \xi(u)}\right)}=\lim _{u \rightarrow \infty} \frac{C^{\prime}\left(p^{*}(u)\right)}{u C\left(p^{*}(u)\right)}=0 .
$$


As Equation (39) holds true for all $u>0,42$ shows that

$$
\lim _{u \rightarrow \infty} \kappa^{\prime}\left(p^{*}(u)\right)=0
$$

Because $p_{\kappa}^{*}$ is the unique positive solution of $\kappa^{\prime}(p)=0$, we then get $\lim _{u \rightarrow \infty} p^{*}(u)=$ $p_{\kappa}^{*}$.

\section{Optimal prevention amount when the adjustment coefficient does not exist}

Let us now consider the case where $X^{(2)}$ follows a sub-exponential distribution and $X^{(1)}$ a light-tailed distribution. This is for example the case when $X^{(1)}$ follows a translated Exponential distribution over the interval $[a, \infty]$ with mean $a+\frac{1}{\lambda}$ and $X^{(2)}$ a Pareto distribution of parameters $a$ and $b$. Then, the stochastic inequality $X^{(1)} \preceq_{\mathrm{hmrl}} X^{(2)}$ holds true when $b \leq \lambda a+1$, as proved in Proposition 2

In such a situation, the moment generating function $M_{X}($.$) does not exist any-$ more. However, we can still determine the optimal prevention amount when $u$ goes to infinity, as shown in the next proposition.

Proposition 8. If Condition (19) holds true, if $X^{(1)} \preceq_{h m r l} X^{(2)}$ and if $D(p)$ follows a sub-exponential distribution for all $p$, then $\lim _{u \rightarrow \infty} p^{*}(u)=p_{\infty}>0$, where $p_{\infty}$ is solution of the equation

$$
-\lambda_{2}^{\prime}(p)\left[\frac{\mu_{2}}{\varphi(0, p)}+\frac{\lambda_{1} \mu_{1}}{\lambda_{2}(p)}\right]=\frac{\lambda_{2}(p) \mu_{2}+\lambda_{1} \mu_{1}}{\varphi(0, p)(c-p)} .
$$

Proof. Proposition 5 guarantees the existence of an optimal prevention amount for all $u$. As seen in (26), we have

$$
\begin{aligned}
\frac{\partial \psi(u, p)}{\partial p}= & \left.\frac{\partial \varphi(0, p)}{\partial p} \sum_{n=0}^{\infty}(1-\varphi(0, p))^{n} \bar{F}_{D(p)}^{* n}(u)\right)\left(1-\frac{\varphi(0, p)}{1-\varphi(0, p)} n\right) \\
& +\varphi(0, p) \sum_{n=0}^{\infty}(1-\varphi(0, p))^{n} \frac{\partial \bar{F}_{D(p)}^{* n}(u)}{\partial p} .
\end{aligned}
$$

Moreover,

$$
F_{D(p)}(u)=\frac{1}{\lambda_{1} \mu_{1}+\lambda_{2}(p) \mu_{2}} \int_{0}^{u} \lambda_{1} \bar{F}_{X^{(1)}}(t)+\lambda_{2}(p) \bar{F}_{X^{(2)}}(t) d t,
$$


which yields

$$
\frac{\partial F_{D(p)}(u)}{\partial p}=\frac{\lambda_{2}^{\prime}(p) \lambda_{1} \mu_{1}}{\lambda_{2}(p)\left(\lambda_{1} \mu_{1}+\lambda_{2}(p) \mu_{2}\right)}\left(F_{D(p)}(u)-F_{D_{X^{(1)}}}(u)\right)
$$

with $F_{D_{X^{(1)}}}(u)=\frac{1}{\mu_{1}} \int_{0}^{u} \bar{F}_{X^{(1)}}(t) d t$.

Also, we have

$$
\frac{\partial \frac{F_{D(p)}^{* n}(u)}{F_{D(p)}(u)}}{\partial p}=\frac{\frac{\partial F_{D(p)}^{* n}(u)}{\partial p} F_{D(p)}(u)-\frac{\partial F_{D(p)}(u)}{\partial p} F_{D(p)}^{* n}(u)}{F_{D(p)}^{2}(u)},
$$

which leads to

$$
\frac{\frac{\partial F_{D(p)}^{* n}(u)}{\partial p}}{F_{D(p)}(u)}=\frac{\frac{\partial \frac{F_{D(p)}^{* n}(u)}{F_{D(p)}(u)}}{\partial p} F_{D(p)}^{2}(u)+\frac{\partial F_{D(p)}(u)}{\partial p} F_{D(p)}^{* n}(u)}{F_{D(p)}^{2}(u)} .
$$

Hence, combining (44), 46) and (48) and letting $u$ go to infinity, we finally obtain

$$
\begin{aligned}
\lim _{u \rightarrow \infty} \frac{\frac{\partial \psi(u, p)}{\partial p}}{\bar{F}_{D(p)}(u)}= & \frac{\partial \varphi(0, p)}{\partial p} \sum_{n=0}^{\infty}(1-\varphi(0, p))^{n} n-\frac{\partial \varphi(0, p)}{\partial p} \sum_{n=0}^{\infty}(1-\varphi(0, p))^{n-1} n^{2} \varphi(0, p) \\
& +\varphi(0, p) \frac{\lambda_{2}^{\prime}(p) \lambda_{1} \mu_{1}}{\lambda_{2}(p)\left(\lambda_{1} \mu_{1}+\lambda_{2}(p) \mu_{2}\right)} \sum_{n=1}^{\infty}(1-\varphi(0, p))^{n} n \\
= & -\frac{\frac{\partial \varphi(0, p)}{\partial p}}{\varphi(0, p)^{2}}+\frac{\lambda_{2}^{\prime}(p) \lambda_{1} \mu_{1}}{\lambda_{2}(p)\left(\lambda_{1} \mu_{1}+\lambda_{2}(p) \mu_{2}\right)} \frac{(1-\varphi(0, p))}{\varphi(0, p)}
\end{aligned}
$$

Now, taking $\frac{\partial \psi(u, p)}{\partial p}=0$ gives the announced result.

\section{Conclusion}

In this paper, we have proposed a risk model with two types of risks where prevention reduces the claim intensity of the most severe risk. In this context, we have studied the ruin probability as a function of the prevention effort. A sufficient condition for prevention to be efficient has been provided, and both cases of light and heavy-tailed claims have been considered. Moreover, we have shown that an insurer should invest more in prevention when its initial reserves are large enough. 
Finally, let us notice that it would be interesting to consider a model where the impact of prevention is not constant over time and where the uncertainty on prevention efficiency is taken into account. This is left for further research.

Acknowledgements : This paper was realized within the framework of the Chair Prevent'Horizon, supported by the risk foundation Louis Bachelier and in partnership with Claude Bernard Lyon 1 University, Actuaris, AG2R La Mondiale, G2S, Covea, Groupama Gan Vie, Groupe Pasteur Mutualité, Harmonie Mutuelle, Humanis Prévoyance and La Mutuelle Générale.

Declaration of interest : Romain Gauchon is employed by addactis France. The paper has been supported by the Chair Prevent'Horizon.

\section{References}

Asmussen, S., Albrecher, H., 2010. Ruin probabilities. volume 14. World scientific.

Bowers, N.L., Gerber, H., Hickman, J., Jones, D., Nesbitt, C., 1984. Actuarial mathematics.

Courbage, C., 2001. Self-insurance, self-protection and market insurance within the dual theory of choice. The GENEVA Papers on Risk and InsuranceTheory $26,43-56$.

Courbage, C., Loubergé, H., Peter, R., 2017. Optimal prevention for multiple risks. Journal of risk and insurance 84, 899-922.

Courbage, C., Rey, B., 2012. Optimal prevention and other risks in a two-period model. Mathematical Social Sciences 63, 213-217.

Cramér, H., 1955. Collective risk theory: A survey of the theory from the point of view of the theory of stochastic processes. Nordiska bokhandeln.

Dickson, D.C., Gray, J., 1984. Exact solutions for ruin probability in the presence of an absorbing upper barrier. Scandinavian Actuarial Journal 1984, $174-186$. 
Dionne, G., Eeckhoudt, L., 1985. Self-insurance, self-protection and increased risk aversion. Economics Letters 17, 39-42.

Dufresne, F., Gerber, H.U., 1988. The probability and severity of ruin for combinations of exponential claim amount distributions and their translations. Insurance: Mathematics and Economics 7, 75-80.

Ehrlich, I., Becker, G.S., 1972. Market insurance, self-insurance, and selfprotection. Journal of political Economy 80, 623-648.

Gauchon, R., Loisel, S., Rullière, J.L., Trufin, J., 2020. Optimal prevention strategies in the classical risk model. Insurance: Mathematics and Economics 91, 202-208.

Gerber, H.U., Goovaerts, M.J., Kaas, R., 1987. On the probability and severity of ruin. Astin Bulletin 17, 151-163.

Heilmann, W.R., Schröter, K.J., 1991. Orderings of risks and their actuarial applications. Lecture Notes-Monograph Series , 157-173.

Hofmann, A., Peter, R., 2015. Multivariate prevention decisions: Safe today or sorry tomorrow? Economics Letters 128, 51-53.

Shaked, M., Shanthikumar, J.G., 2007. Stochastic orders. Springer Science \& Business Media. 